\title{
Method for Extracting True Stress from TEM in situ Compression Testing
}

\author{
Haozheng Qu ${ }^{1}$, Kayla H. Yano ${ }^{2}$, Priyam V. Patki ${ }^{3}$, and Janelle P. Wharry ${ }^{3}$ \\ 1. School of Mechanical Engineering, Purdue University, West Lafayette, Indiana, USA \\ 2. School of Materials Engineering, Purdue University, West Lafayette, Indiana, USA \\ 3. School of Nuclear Engineering, Purdue University, West Lafayette, Indiana, USA
}

Transmission electron microscopic (TEM) in situ mechanical testing approaches are increasingly being used to investigate plastic behaviors of materials at the nanoscale, especially in thin films, implanted or irradiated specimens, and other volume-limited materials. Recent results from depth-sensing TEM in situ compression pillar tests have shown the method is capable of providing meaningful quantitative measurement of yield strength and elastic modulus of specimens with low characteristic length (i.e. high number density of obstacles to dislocation motion) [1-3]. However, data analytics for these tests remain in their infancy. Tests output is typically comprised of the load and displacement at each time step. The researcher must then know the specimen dimensions in order to generate a stress-strain curve, from which yield stress can then be determined. In all reports in the archival literature, these are engineering stress-strain curves - i.e., the original specimen dimensions are used to calculate stress from load. However, since nearly all materials exhibit a nonzero Poisson's ratio, most compression pillars will exhibit "barreling" during deformation. In such cases, the true stress-strain relationship may be of greater interest than the engineering stress-strain curve.

Obtaining a true stress-strain curve is easier said than done, however. Particularly, it requires precise knowledge of the specimen dimensions at every time step during the deformation experiment. While this could theoretically be obtained by manually measuring the specimen dimensions during each frame of the compression test video, this would be an unreasonably tedious task, as each test video contains hundreds to thousands of frames. Instead, we herein develop an algorithm to automatically extract specimen dimensions from compression pillars. We utilize the algorithm to generate true stress-strain curves and calculate the strain hardening exponent. This algorithm can be utilized on both TEM and scanning electron microscopic (SEM) in situ compression test videos.

We carry out the true stress-strain analysis on two alloys for which we have already conducted and published TEM in situ compression pillar results. First is a model Fe-9Cr oxide dispersion strengthened (ODS) alloy, irradiated with $5 \mathrm{MeV} \mathrm{Fe}^{2+}$ ions to 3 displacements per atom (dpa) at $500^{\circ} \mathrm{C}$ [2]. Second is a nanocrystalline $\mathrm{Cu}-10 \mathrm{Ta}$ alloy containing a bimodal Ta-rich nanophase distribution, irradiated with 2 $\mathrm{MeV}$ protons to $1 \mathrm{dpa}$ at $500^{\circ} \mathrm{C}[3]$.

The process is designed to automate the calculation of the real-time compression pillar width; together with load and displacement data from the experiment, the process then calculates the true stress-strain curve and extracts the strain hardening exponent. There are two components to the overall process: (1) a width tracking algorithm, and (2) true stress calculator. The width tracking algorithm, is a function script written in Matlab ${ }^{\mathrm{TM}}$, which converts each frame of the video to greyscale for mathematical filtering. In greyscale, the TEM vacuum is represented as blank (i.e. 0) in the logical map of the graph. Two starting points (marked as 'o' and ' + ' in Figure 1a) are set by passing through a logical filter that identifies the points with all-blank (i.e. all 0) surroundings on the left and right sides of the image. These starting points ensure that the pillar edge tracking begins in the TEM vacuum, rather than on the specimen or 
compression head. From these starting points, the algorithm moves toward the center of the image until it encounters the pillar edges; these edges are marked with a cross (' $x$ ' symbol in Figure 1a). The algorithm moves onto the next line in the logical map until it marks five points (or more based on user preference), or until it reaches the upper or lower boundary of the TEM vacuum. After five pairs of edge points are marked, the distance between each pair is calculated, and the average of these distances is exported as the output of the tracking algorithm.

The second component of the approach calculates true stress and strain hardening exponent. The raw load-displacement data obtained from the experiment is read in to Matlab ${ }^{\mathrm{TM}}$. The user specifies the pillar height, and the pillar widths (as a function of time) are called from the tracking algorithm. The true stress and strain are then calculated at each time step, based on the real-time pillar dimensions. True stress and true strain are output as an array, which the user can subsequently plot. Then, to determine the strain hardening exponent, true stress and strain are converted to logarithmic scales, and the slope of the linear portion of the curve is calculated using linear regression. For the Fe-9Cr ODS irradiated with 5 $\mathrm{MeV} \mathrm{Fe}^{2+}$ ions to $3 \mathrm{dpa}$ at $500^{\circ} \mathrm{C}$, the strain hardening exponent is determined from TEM in situ compression to be 0.493 . This value is nearly double that measured on the same alloy by nanoindentation, 0.24-0.33 [4]. This is unsurprising, because reports have shown that the intermittent plastic flow of nano- to micro-scale specimens inhibits a meaningful strain hardening rate from being determined from these tests [5].

(a)

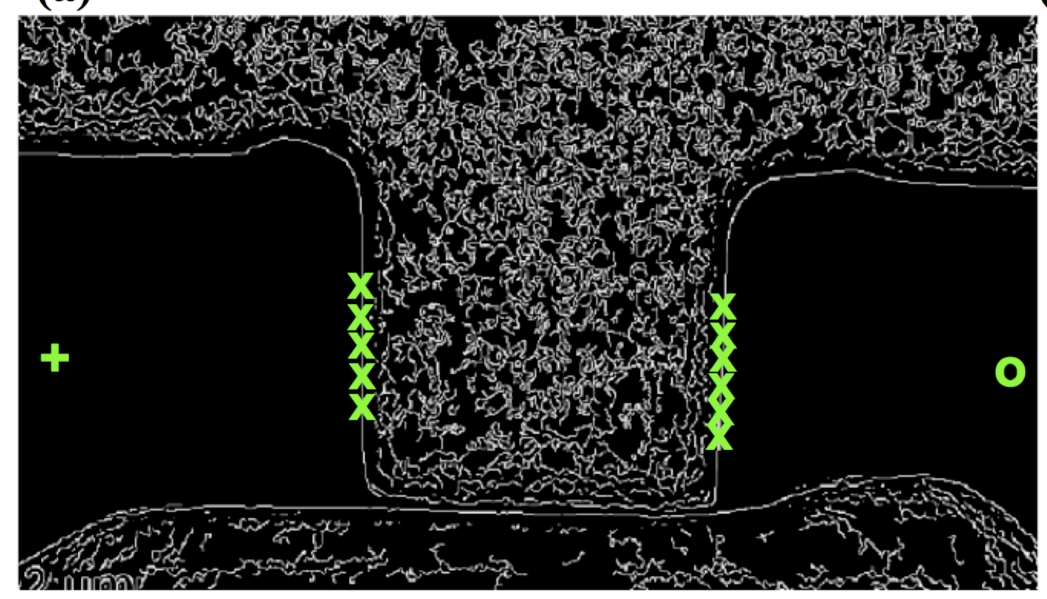

(b)

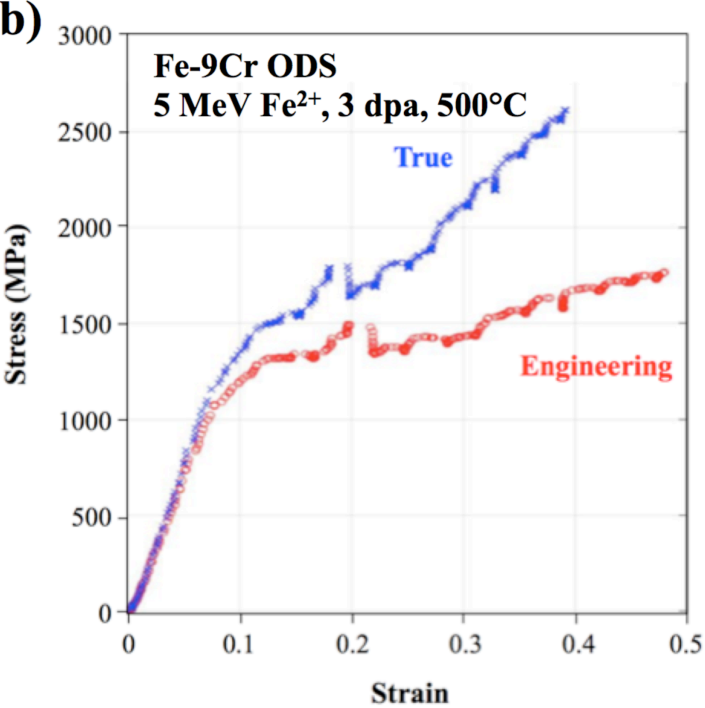

Figure 1. (a) Example still-frame from greyscale image in algorithm, showing starting points $(0,+)$ and edge identification (marked by ' $\mathrm{x}$ '); (b) engineering compared to true stress-strain curves from $\mathrm{Fe}-9 \mathrm{Cr}$ ODS, irradiated with $5 \mathrm{MeV} \mathrm{Fe}^{2+}$ ions to $3 \mathrm{dpa}$ at $500^{\circ} \mathrm{C}$.

References:

[1] D. Kiener, et al., Nature Materials 10 (2011) 608-613.

[2] K.H. Yano, et al., J Nuclear Materials 483 (2017) 107-120.

[3] K.H. Yano, et al., Transactions of the American Nuclear Society 116 (2017) 22103.

[4] C.K. Dolph, et al., J Nuclear Materials 481 (2016) 33.

[5] D. Kiener, C. Motz, and G. Dehm, Materials Science \& Engr A 505 (2009) 79-87.

[6] The authors acknowledge funding from the Nuclear Regulatory Commission grant NRC-HQ-84-14G-0056, the US DOE Nuclear Science User Facilities experiment 15-540, and Purdue University. 